\title{
LES MÉDIAS EN AFRIQUE SUBSAHARIENNE : UNE APPROCHE EN TERMES D'USAGES
}

\author{
Usages des radios dans la citoyenneté \\ au Burkina Faso
}

\author{
Sylvie Capitant ${ }^{1}$
}

Les médias africains ne sont pas à proprement parler les parents pauvres de la sociologie africaniste. Dès les années 50, une littérature conséquente commence de paraître mais c'est véritablement dans les années 90 avec la « démocratisation » que connaît la région, que cette littérature prendra toute son ampleur. Les angles d'approche possibles sont nombreux : impact, rôle ou fonction des médias ? Médias africains comme partenaires de développement, de démocratie, de guerre ou de paix ? Pour développer son travail, un chercheur, tel un alpiniste escaladant une paroi rocheuse, doit évaluer et choisir un « couloir d'escalade » menant à son sujet. Née d'une réflexion sur la place des médias dans la citoyenneté en Afrique susbsaharienne, la recherche, dont cet article rend compte, a du se plier à ce travail de repérage et choisir un

1 IEDES- La Sorbonne Paris I

Recherches en communication, $\mathrm{n}^{\circ} 26$ (2006). 
« couloir ». Cependant, une revue des approches de la manière dont les médias africains ont été étudiés jusque là laisse entrevoir deux particularités majeures. Tout d'abord, la proposition théorique a été faible. Contrairement à la sociologie des médias générale qui a produit de nombreuses théories, celle des effets limités, celle du lecteur-récepteur, celle des médias comme outils de domination, la sociologie des médias en Afrique s'est laissée enfermée dans la recherche empirique, envisageant, selon les modifications socio-politiques de la sous-région, les médias comme des multiplicateurs de développement, des outils de domination du Nord, des outils de démocratie et plus récemment comme des outils de guerre ou de paix. L'autre faiblesse de ce domaine de recherche est sa tendance à vouloir se construire en marge de la sociologie des médias centrale, pour reprendre le terme de P. Quantin. Ce dernier, réfléchissant sur l'étude du vote en Afrique, constate que les processus électoraux africains ne semblent pas dignes d'intégrer la « science politique centrale ». Au nom d'une appartenance à une certaine aire culturelle, les études sur la question ont développé une « science politique périphérique » qui répond à d'autres principes que ceux développés dans la science politique générale. Quantin propose ainsi de «banaliser l'approche du politique africain ». (QUANTIN PATRICK 2005) Il semble que cette dichotomie existe aussi dans l'étude des médias en Afrique.

Faisant cas de ces deux particularités soulignées, cet article entend proposer de réintégrer l'étude des médias en Afrique à la sociologie des médias générale. Il s'agit d'interpeller les concepts théoriques proposés par cette dernière afin de construire des études plus problématisées et moins empiriques des médias africains. Ce choix scientifique, dont on envisagera les enjeux, permet d'établir une nouvelle approche des médias en Afrique. Nourrie de la théorie des usages et des gratifications de Katz et de la sociologie des publics qui occupe une place centrale aujourd'hui dans les problèmes de communication, une approche des médias africains en termes d'usages sera ainsi proposée. Cette approche a été développée dans le cadre d'un travail de doctorat portant sur les usages des radios au Burkina Faso dans la citoyenneté dont il sera rapidement rendu compte ici. 


\section{L'étude des médias en Afrique : un domaine de recherche périphérique?}

\section{Revues des approches}

Même si des médias de masse existent en Afrique depuis la fin du XIXème siècle ${ }^{1}$, les premières études les concernant datent des années 40. Mais c'est au moment des indépendances qu'un premier paradigme apparait. Considérant à l'époque le Tiers Monde comme des sociétés traditionnelles « en retard économiquement », Rostow envisage pour lui les différentes « étapes de la croissance économique » qui débute par la phase du « décollage économique ». Dans cette perspective, les médias africains vont apparaître comme des outils de modernisation et comme des «multiplicateurs de développement». Pour Lerner qui envisage la modernisation comme «le processus séculaire de changement vers un système social de participation » (LERNER DAVID 1958), les médias constituent une des variables de modernisation par leur influence sur le taux d'alphabétisation et sur la participation politique. Ces idées seront reconnues officiellement par l'Unesco qui chargera William Shramm de réfléchir sur le lien entre médias et développement dont il rendre compte dans Mass Media and National Developpement (SCHRAMM WILLIAM 1964). De même, les dirigeants africains appuieront cette idée et, souvent pour des raisons politiques, imposeront un monopole étatique sur les médias de masse afin que ces derniers remplissent leur mission de développement et de construction d'un sentiment national.

Dans les années 70 le discours se modifie, sous l'influence de la pensée critique d'inspiration marxiste qui influe les sciences sociales dans leur ensemble. Le Tiers Monde n'est plus vu sous le prisme tradition/modernité mais sous celui de la domination. L'Occident est au centre d'une aire de domination à profil concentrique dans laquelle le Tiers Monde n'est qu'une périphérie exploitée par le centre. Les pays non alignés en appellent à une nouvel ordre mondial plus équilibré. $\mathrm{Au}$ fur et à mesure, les questions de communication vont prendre une

1 Le premier journal d'Afrique date de 1800 année où est lancé « Cap Town Gazette » destiné aux Européens du Cap en Afrique du Sud. Le premier journal africain date de 1826 au Liberia, voir TUDESQ André-Jean. Journaux et radios en Afrique aux XIXe et XXe siècles, Paris : Groupe de recherche et d'échanges technologiques (1998). 
place de plus en plus importante. Lors de la conférence de Nairobi en 1974, organisée par l'Unesco, les pays non alignés exigent un NOMIC, un nouvel ordre mondial de l'information et de la communication. Les pays du Nord aliènent les pays du Sud par une production excessive vue comme une volonté de colonisation culturelle. Ce nouveau paradigme va entraîner les premières recherches sur la réception en Afrique : quels sont les effets socio-culturels des programmes occidentaux sur les cultures du Sud ? Quelle la compréhension au Sud de ces programmes? Cependant, les recherches empiriques seront peu nombreuses, et le paradigme critique sera plus virulent sous la forme de déclaration que d'enquête empirique. Cette vision sera cependant adoubée par l'Unesco qui publie en 1980 le rapport Mac Bride, donnant un grand écho à ces idées.

Mais c'est au cours des années 1990 que la littérature sur les médias en Afrique connaît son véritable foisonnement. Une étude bibliographique laisse voir que $80 \%$ des ouvrages portant sur le thème Médias et Afrique sont postérieurs à 1990. L'Afrique subsaharienne connaît à cette période de profonds changements politiques : avènement du multipartisme, libéralisation politique, libéralisation médiatique, rédaction de constitution reconnaissant le vote pluraliste comme mode de désignation des dirigeants. En quelques années, voire parfois en quelques mois, l'Afrique se construit un arsenal juridique et constitutionnel conforme au système institutionnel démocratique. Les années passant prouveront que les changements institutionnels n'ont pas toujours entraîné un changement de pratiques politiques. Toujours est-il que dans ces années là, on assiste à une «éclosion médiatique » sous le coup d'une « fièvre démocratique » (FRERE M-S 2000). Entre 1990 et 1993, plus de 50 journaux sont créés au Mali. A partir de 1995, c'est au tour des radios privées de s'ouvrir. Comme le rappelle Tudesq, dans les années 80, dans la sous-région, « il n'y avait que 5 radios indépendants des gouvernements, il y en a actuellement un millier » (TUDESQ ANDRÉ-JEAN 2000). Cette évolution majeure va susciter une profusion de publications qui vont s'articuler autour de trois thèmes. Le premier révèle les médias comme les nouveaux acteurs de la nouvelle société civile africaine. Ils permettent de «publiciser les maux », de « sortir les gouvernements de leur clandestinité et de créer un espace public de discussion » (HYDEN G.; LESLIE, M. 2002). Les médias ont occupé rapidement une place importante dans les recherches sur la société civile africaine car pour beaucoup ils ont rempli « un vide dans l'arène 
politique » (HYDEN GÖRAN; OKIGBO, C. 2002). Contrairement à l'Europe de l'Est et à l'Amérique du sud, ces auteurs considèrent que la société civile en Afrique était faible : peu de syndicats, peu d'associations, des Églises peu engagées etc... Ainsi les médias ont été investis d'une lourde charge : "nourrir le débat démocratique, contribuer à fixer l'agenda de l'évolution du projet démocratique. Ils doivent prévenir les dérapages que ce soit une décrépitude institutionnelle ou une corruption individuelle. Dans le cas où d'autres partis politiques d'opposition manqueraient à constituer une véritable opposition, une telle opposition devrait venir des médias. » (RANDALL VICKY 1993) Le deuxième thème qui structure cette littérature porte sur le pluralisme et l'indépendance des médias. Consacré par la déclaration de Windohek qui appelle au « Développement d'une presse indépendante et pluraliste » (UNESCO 1991), ce thème va entraîner les chercheurs à décrire l'extrême dynamisme du milieu médiatique privé dans les années 90 et les bailleurs de fonds à financer largement ces nouveaux médias. Seulement, progressivement cet enthousiasme s'émousse et les chercheurs soulignent de plus en plus le manque de professionnalisme de la presse et sa tendance à « l'affairisme médiatique » (ADJOVI E. 2003, Déc.) L'implication de la radio RTLM dans la génocide rwandais va profondément influencer la recherche. Un changement s'opère : un média privé n'est pas forcément un média indépendant, le nombre de journaux n'implique par forcément un espace de discussion ouvert et enfin la libéralisation médiatique ne transforme pas nécessairement les médias en outils de démocratie.

Cette désillusion va susciter un nouveau courant de recherche axé sur les conditions d'existence des médias ainsi que sur leurs effets dans un contexte de guerre. Ce domaine, encore relativement nouveau, est encore principalement animé par les opérateurs de développement ${ }^{1}$. Ces derniers tendent à vouloir faire des médias des « médiateurs » en situation de conflits. On parle désormais de « journalisme de paix », de «journaliste pro-actif » ou encore de « journalisme sensible au conflit ». (HOWARD ROSS. 2005).

Cette revue des approches révèle que les médias en Afrique ont tour à tour été considérés comme des outils de développement, des

1 Selon Howard Ross, 69 organisations intègrent les médias dans leurs interventions de règlement des conflits. 
outils de domination, des outils de démocratie et enfin des outils de paix. Cette évolution montre à quel point l'étude des médias a été tributaire des évolutions de la perception des pays de sud successivement vus comme en phase de modernisation économique et sociale, comme une zone périphérique de domination du Nord, comme une zone de démocratisation et enfin aujourd'hui comme une zone de troubles où conflits et restaurations autoritaires, pour reprendre le terme de J.F. Bayart, sont en cours. Cette évolution circonstancielle, certes nécessaire pour produire des recherches pertinentes, pose cependant le problème de l'apport théorique de cette littérature. Quelles sont les constructions théoriques de cette littérature et quelle sont leur place au sein de la sociologie générale des médias?

\section{Un domaine de recherche périphérique}

Comme il a été souligné en introduction, et comme la revue des approches le laisse entrevoir, l'étude des médias en Afrique est caractérisée par une faible approche théorique. Même si des thèmes ou des axes de recherche ont été développés, peu de postulats plus généraux ont été formulés à l'exception de quelques chercheurs anglophones. Hyden et Leslie formulent ainsi que l'impact des médias sur la démocratie ne peut pas être bien appréhendé si on ne distingue pas quatre domaines spécifiques : politique, technologique, économique et culturel. Dans le domaine politique, l'influence des médias se mesure en fonction du degré de contrôle de l'État, du niveau de représentation des médias (qui accède aux médias et qui s'y reconnaît) et enfin le degré de connexion internationale de ces médias (HYDEN G.; LESLIE, M. 2002). Ces tentatives de théorisation sont rares et même sur le thème médias et démocratie, qui a beaucoup occupé les chercheurs, R. de La Brosse admet que si « le rôle des médias en tant qu'acteurs des transitions démocratiques est clairement affirmé par les chercheurs » jusqu'à apparaître comme « un dogme incontestable », c'est « toujours sans parvenir à en exprimer la complexité et la réalité ». Le mode d'influence des médias sur les processus politiques reste flou et « aucune articulation systématique du rôle des médias » n'a été proposée (DE LA BROSSE RENAUD 1999 ; 2002).

L'étude des médias en Afrique est aussi caractérisée par une absence de référence aux concepts de la sociologie des médias. Les modèles de cette dernière sont nombreux : théorie des effets limités de 
Lazarsfeld, la modèle des fonctions de Wright, l'approche des usages et des gratifications de Katz et Gurevitch, la théorie de l'agenda de Mc Combs et Shaw, la pensée critique héritière de l'école de Francfort, le modèle du texte-lecteur et des lectures négociées des Cultral Studies, les théories de la réception, les approches en termes d'espace public, et enfin la sociologie des publics de Cerfaï et Pasquier. Aucune étude portant sur les médias en Afrique ne revendique une parenté avec ces théories. Pourtant des liens peuvent être dégagés : les réflexions autour des médias comme outils de démocratie ont clairement été influencées par les théories de l'espace public, les médias comme outils de domination font écho à la pensée d'Althusser pour qui les médias de masse sont des «appareils idéoliques d'État» (ALTHUSSER LOUIS 1970). Mais ces liens ne sont jamais revendiqués ni affirmés. Comme si la recherche sur les médias en Afrique ne pouvait se servir des apports de la sociologie des médias du Nord. Les causes de ce particularisme entretenu sont nombreuses et ne pourront faire ici l'objet d'une étude approfondie. Signalons seulement le poids des aires culturelles dans le domaine académique, la contradiction entre sous-médiatisation en Afrique et sur-médiatisation en Occident ou encore l'idée que l'opinion publique relève d'un processus individuel supposant l'usage individuel de sa raison et qu'en Afrique on considère toujours que la société holiste ne permet pas ce genre de développement. Il demeure cependant que les études des médias en Afrique, par un particularisme développé, se sont construites comme un domaine d'étude « périphérique » plus rattaché au milieu africaniste qu'au milieu de la communication. Les revues de diffusion de cette littérature en témoignent: Politique Africaine, Afrique Contemporaine, Journal des Africanistes, Monde en développement. À l'exception tout de même d'Hermès qui en 2000 a diffusé un numéro comprenant une large partie sur les médias africains ${ }^{1}$. Une forte dichotomie donc qui pose la question d'une éventuelle convergence.

\section{Une nécessaire convergence}

Les arguments en faveur d'une convergence sont de trois ordres. En premier lieu, les paysages médiatiques africain et européen partagent de plus en en plus de points communs. Médias et démocratie est un des thèmes centraux de l'étude des médias en Afrique. Or, cette ques-

1 Amérique Latine et Communications . Hermès, 28, (2000), Rubrique Varia, pp. 207270. 
tion s'avère de plus en plus pertinente dans le contexte européen. La presse européenne est confrontée à un sérieux problème d'indépendance financière. Concurrencée par les médias sur internet et par une presse gratuite, nombre de commentateurs constatent que la presse, notamment en France, est entre les mains de quelques grands groupes financiers. On retrouve là un des grands thèmes de la littérature sur les médias en Afrique : indépendance et pluralisme. Le deuxième argument porte sur la nature de l'opinion publique en Afrique considérée trop souvent relevant d'un mode d'élaboration traditionnelle sur lequel les médias ne pourraient avoir aucune prise. Certains exemples historiques montrent cependant le contraire. Les observateurs des mouvements de démocratisation, bien qu'ils reconnaissent le poids des facteurs externes dans ce processus, soulignent cependant avec force l'importance qu'a pu avoir la contestation interne. Cette contestation, nourrie de l'effondrement du système de parti unique mis en place depuis les indépendances, a beaucoup été influencée par les images diffusées par les télévisions sur le procès de Ceausecu, la fin des régimes autoritaires communistes et la chute du mur de Berlin (DE LA BROSSE RENAUD 1999 ; HYDEN GÖRAN; OKIGBO, C. 2002). Le troisième argument en faveur d'une convergence qui peut être ébauché ici concerne le manque d'appui théorique des études sur les médias en Afrique. Cette insuffisance théorique peut s'expliquer par une volonté de ne pas faire appel aux outils d'une discipline dont on refuse la filiation. Pourquoi par exemple les nombreuses études sur la réception qui sont produites aujourd'hui en sociologie des médias et qui posent la question du public n'ont donné lieu qu'à peu de développement en Afrique ? Par exemple, l'ouvrage pourtant brillant de M.S. Frère sur le discours de la presse dans les transitions au Niger et au Bénin ne mentionne à aucun moment que seuls $10 \%$ de la population est capable de lire la presse dont elle décortique les mots (FRERE M-S 2000). Comme le souligne Balima à propos de l'Afrique «la question du public, donc de la réception, est l'une des plus importantes, mais elle est restée longtemps peu analysée. [...] Elle suscitait moins de curiosité que les réformes institutionnelles ou administratives. [...] Comment d'un côté admettre l'intelligence des citoyens au point d'en faire la source de la légitimité démocratique et de l'autre supposer les publics négligeables parce qu'ils sont influençables et manipulables ? 》(BALIMA SERGE-THÉOPHILE 2000) Ce décrochage théorique sur la question du public et de sa réception s'observe aussi sur les questions de la publicisation des problèmes publics, sur l'analyse des contextes de réception, sur le lien entre médias et mobi- 
lisation, enjeux qui sont cœur des débats contemporains mais qui ne donnent lieu qu'à des développements timides dans le contexte africain. Cette dichotomie conduit à des recherches descriptives et peu problématisées des médias en Afrique, car il leur manque un socle théorique.

L'idée est donc de soutenir une convergence entre la sociologie des médias et l'étude des médias en Afrique. Non pas sous la forme d'une transposition d'un contexte à l'autre mais sous la forme d'une transcription. Les concepts développés dans une perspective européenne de sur-médiatisation, d'accès croissant aux nouvelles technologies de l'information et de la communication, de société civile puissante et hétéroclite, ne peuvent s'appliquer tels quels dans le contexte africain. Seulement, pourquoi ne pas s'inspirer des nouvelles approches pour construire une méthodologie plus systématique de compréhension des médias africains ? Pourquoi ne pas développer les approches de réception en Afrique, quitte à transformer les concepts premiers ou à en produire d'autres ? C'est l'objet de l'approche en termes d'usages proposée ici. Elle fait appel à la théorie des usages et des gratifications de Katz et Gurevitch ainsi qu'à la sociologie des publics. Elle a été transcrite dans le cadre d'une étude sur l'usage des radios dans la citoyenneté au Burkina Faso.

\section{Une approche en termes d'usages}

Une approche en termes d'usages place le public au centre de son analyse selon un modèle circulaire de communication proposée par Wiener et réactualisé par la sociologie interactionniste de Goffman, Birdwhistell et Bateson. L'accent n'est pas mis sur le message mais sur le contexte des processus communicationnels médiatiques conçus comme une interaction permanente entre l'émetteur et le récepteur qui non seulement est co-producteur du message mais qui, de plus, use du message dans ses sphères de sociabilité qu'elle soient interpersonnelles ou publiques. Dans la perspective d'une étude sur la citoyenneté en Afrique subsaharienne, l'idée ne sera pas d'étudier ce que les médias disent sur la démocratie, mais d'évaluer si les individus usent des médias pour réaliser leur citoyenneté ou si c'est le cas, de quelle manière ils en font usage. La réalisation de la citoyenneté sera comprise comme l'actualisation de six pratiques démocratiques : pratiques de décision, d'expression, de réunion, de protestation, de délibération et de participation. 
Cette approche fait à appel à la théories des usages et des gratifications ainsi qu'à la sociologie des publics.

\section{Renouvellements théoriques}

Dans les années 70, Katz et Gurevitch, inscrits dans le courant de la recherche empirique américaine, proposent d'évaluer la question de l'effet des médias selon une approche dite des usages et des gratifications. Leur intuition première est de considérer le récepteur comme un sujet actif qui use des médias plus qu'il ne les subit. À cette époque, l'idée est assez novatrice. Ensuite, poursuivant la théorie fonctionnaliste, ils considèrent que les médias aident les récepteurs à satisfaire des besoins sociaux importants. Ils en identifient 35 comme le besoin de s'informer sur l'action du gouvernement ou encore le besoin de se distraire etc... L'idée est stimulante car le mouvement part du récepteur, c'est lui qui use d'un média pour satisfaire un besoin. De plus, cet angle d'observation permet de mettre en concurrence les médias avec d'autres outils. Le modèle n'enferme pas le récepteur dans un tête-à-tête irréel avec le média et cherche à comprendre ce que font les récepteurs des médias et non pas ce que les médias font aux récepteurs. Leurs conclusions soulignent que les médias sont modérément utilisés par les récepteurs qui en général disent préférer d'autres moyens que les moyens médiatiques, comme les relations interpersonnelles, pour satisfaire leurs besoins importants. Cette approche a été critiquée, notamment par Ien Ang, pour sa trop forte prégnance psychologique et individuelle. (ANG I 1990). Il n'en demeure pas moins que la méthodologie est intéressante par le rôle actif qu'elle donne au récepteur, par la mise en concurrence des médias avec d'autres outils et par une approche de la réception, non pas seulement comme un décodage de texte, mais comme une activité dynamique qui se réalise dans un contexte particulier et qui ouvre la porte à des activités de socialisation. Enfin, comme le soulignent les auteurs, elle a été pensée pour « servir de base à l'extrapolation et à la comparaison interculturelle » (KATZ ELIHU ; GUREVITCH, MICHAEL; HAAS, HADASSAH 1973).

L'autre source d'inspiration de l'approche en termes d'usages proposée ici est la sociologie des publics dont Cerfaï et Pasquier rendent compte dans l'ouvrage Les sens du public (CERFAÏ DANIEL ; PASQUIER, DOMINIQUE. 2003). Pour les auteurs, le public n'est pas une donnée objective, il est dynamique et émerge dans certains 
contextes. Ils soutiennent que les études sur le public doivent s'orienter vers l'étude des expériences publiques. Les publics sont engagés dans des « registres d'expériences » qu'on doit analyser selon « une pragmatique des activités ». Il s'agit « d'examiner des performances en train de se faire en situation et des contraintes écologiques qui pèsent sur ces pratiques ». La sociologie des publics est une sociologie des contextes attentive aux situations d'émergence de la vie collective. Elle va chercher à comprendre les processus de réception et ce qu'ils impliquent comme mobilisation des ressources individuelles. Mais surtout, ce qui est plus nouveau, elle tente de déterminer ce que deviennent les interprétations faites des messages dans les interactions quotidiennes à travers « tous les lieux où les expériences singulières se transforment en engagement collectif » (CERFAÏ DANIEL ; PASQUIER, DOMINIQUE, 2003) ou pour reprendre la belle formulation d'Eliasoph à étudier « la parole politique telle qu'elle circule et se temporalise dans les différentes provinces de la vie quotidienne » (ELIASOPH N. 2003).

\section{De l'usage des médias dans la citoyenneté en Afrique et de leur mesure}

Héritière de ces deux théories, l'approche en termes d'usages s'appuie sur les bases suivantes :

- le public est au centre de l'analyse;

- la réception est comprise non pas seulement comme l'interprétation des messages médiatiques mais comme l'usage que les publics font de ces messages;

- les outils médiatiques sont placés en concurrence avec d'autres outils;

- l'accent est mis sur les contextes et les espaces où se temporalisent l'usage des messages médiatiques.

Développée dans le cadre d'une réflexion initiale sur les médias et la démocratie en Afrique subsaharienne, cette approche en termes d'usages s'est révélée pertinente. L'approche de la démocratie en Afrique a souvent été biaisée par une approche trop institutionnelle, négligeant l'ambivalence du terme démocratie qui désigne à la fois un type de régime politique (caractérisé par des élections concurrentielle, le mutlipartisme, la liberté d'expression etc...) et un idéal politique dont John Dewey ou Benjamin Barber par exemple ont défendu une vision exigeante (BARBER BENJAMIN 1997 ; DEWEY J. 1927). Ainsi le vent 
de démocratie qu'on a dit souffler en Afrique subsaharienne s'est suivi d'une certaine désillusion qui a même fait crier à certains $A$ bas la Démocratie (N'GORAN KWAME 2001). En effet, malgré des constitutions, l'existence de plusieurs partis, une certaine liberté de la presse, les alternances n'ont pas été nombreuses dans la sous-région. Certains dirigeants en ont même profité pour « démocratiser » leur pouvoir à vie, modifiant la constitution pour pouvoir rester légalement au pouvoir. La démocratie se différencie selon qu'on l'étudie selon le texte ou l'esprit. Aussi vouloir étudier l'impact des médias sur la démocratie pose problème tant il est difficile de proposer une définition pertinente et opératoire de ce terme. La notion de citoyen peu alors être mobilisée, mais il apparaît rapidement que le terme citoyen renferme la même ambivalence que la démocratie. Il est lui aussi partagé entre une vision forte qui fait de lui la source de la légitimité politique et l'acteur central de la sphère publique et une vision plus institutionnelle qui le cantonne à son droit de voter et qui tend à le considérer comme l'équivalent dans le domaine politique du consommateur dans le domaine économique. Les sondages mesurent ainsi les volontés des citoyens mais d'une manière consumériste comme le souligne Patrick Champagne (CHAMPAGNE PATRICK 1995).

Face à ces difficultés définitionnelles, la proposition de Guy Hermet concernant la citoyenneté apporte un éclairage. $\mathrm{Si}$ « la conscience d'un ensemble de droits et de devoirs concédés par l'État » est au cœur de la citoyenneté, cette dernière ne se réalise qu'à l'aide "d'une série de comportements civiques effectifs, qui vont du simple effort d'information sur les problèmes du jour à l'engagement militant en passant par le dépôt d'un bulletin de vote dans l'urne. Sans cela, toutes les constitutions et toutes les déclarations des droits qu'on voudra ne servent à rien » (HERMET GUY 1993). La citoyenneté s'aborde par le biais de ces « comportements » dont Hermet parle, que Eliasoph nomme « pratiques civiques » et que la recherche exposée ici rend compte sous le terme de «pratiques démocratiques ». Cette acception de la citoyenneté a l'avantage de proposer une vision plus pragmatique de la démocratie, et donc plus opératoire. De plus, elle relève le défi de la vision institutionnelle de la démocratie en insistant sur les processus par lesquels la démocratie se réalise et non pas seulement sur une vision légaliste. L'existence institutionnelle de la démocratie ne renseigne pas sur la virulence des pratiques des protestation par exemple. Par exemple en Pologne, lors de la chute du régime communiste, bien que le régime ne soit pas démocratique, les pratiques démocratiques de protestation 
furent nombreuses. Analyser la démocratie par ses pratiques permet de construire un modèle de recherche plus opératoire qui, de plus, se prête plus à la comparaison inter culturelle. En effet, les pratiques s'observent plus qu'elles ne sont construites pas le chercheur, et cela dans différents contextes. Les pratiques de protestation se pratiquent différemment au Burkina Faso, au Pérou ou en France. Les décrire permet de tracer une carte de la nature de la démocratie dans différents pays : quels sont les acteurs, quelle formes de protestation choisissent ils, quels en sont les enjeux etc... Si le cadre institutionnel de la démocratie est sensiblement le même partout, la manière de le pratiquer diffère. Étudier les pratiques démocratiques permet ainsi de rendre compte de la réalité concrète de la démocratie dans différents pays. Pour fournir un cadre d'étude, ces pratiques ont été identifiées au nombre de six : pratiques de décision, d'expression, de réunion, de protestation, de délibération et de participation. Malgré ce cadre formel, la déclinaison des ces pratiques diffère selon les contextes. C'est au chercheur de rendre compte de cette conjugaison.

Aussi réfléchir à l'impact des médias sur la démocratie en Afrique subsaharienne prend plus de pertinence sous la formulation suivante : quel est l'usage des médias dans les pratiques démocratiques en Afrique subsaharienne. En effet, l'approche en termes d'usages se prête facilement à une réflexion sur la citoyenneté. Usages médiatiques et pratiques démocratiques partagent ce même souci des contextes. Pour en offrir un modèle plus opératoire, nous proposons trois manières de mesurer ces usages :

- Usages comme pratiques médiatiques. C'est à dire relever dans un premier temps la manière dont les gens usent de leurs médias : temps d'écoute, lieux d'écoute, quelles radios écoutentils, quelles émissions etc... Une acception relativement classique mais néanmoins nécessaire

- Usages comme saisine. Qui se saisit des médias pour s'exprimer, pour faire passer une idée ? Qui se saisit des médias pour s'informer?

- Usages comme sources d'action publique De quelle manière les médias conduisent leurs publics à s'engager dans le registre des expériences publiques ? Les électeurs se servent ils des médias pour déterminer leur vote ? De quelle manière les médias interagissent sur les engagements dans la sphère publique ? 
Cette approche a été développée dans le cadre d'une recherche sur l'usages des radios dans la citoyenneté au Burkina Faso, dont nous brosserons, pour finir, rapidement la méthodologie.

\section{Le cas des radios au Burkina}

La situation politique et médiatique du Burkina Faso ne sautait être décrite ici par manque de place. Signalons seulement que le Burkina Faso offre une garantie constitutionnelle de tous les droits politiques et civiques, que des élections multipartites sont organisées depuis 1991, que son président Blaise Compaoré est au pouvoir depuis 1987 à la suite d'un coup d'État et qu'il a été élu en 1991, il était alors le seul candidat, puis en 1998 puis en 2005 avec plus de $80 \%$ des suffrages exprimés. La constitution révisée en 2000 limite le nombre de mandats présidentiels à deux. Le conseil constitutionnel a jugé en 2005 que la loi n'étant pas rétroactive, Blaise Compaoré avait le droit de se présenter en 2005 et donc aussi en 2010, le mandat étant devenu un quinquennat. La presse privée a été sur le principe autorisée en 1991, mais le corpus juridique portant création des règles de fonctionnement des médias privés audiovisuels date de 1998. La plupart des radios ont été créées dans les années 95. La presse se compose de 4 quotidiens, d'une dizaine d'hebdomadaires, de 2 bimensuels et d'une dizaine de mensuels. La paysage audiovisuel se compose de 19 radios privées associatives, 19 radios privées commerciales, de 18 radios confessionnelles, 4 radios internationales, de 11 radios locales publiques et d'une radio publique qui est la seule à couvrir presque l'ensemble du territoire national. On compte enfin 1 télévision nationale et 2 télés privées à Ouagadougou, la capitale $^{1}$. La liberté de la presse a été marquée par l'assassinat en 1998 du journaliste Norbert Zongo qui a provoqué un profond séisme politique dans le pays pendant plus de deux ans. Le dossier a finalement été classé en août 2006 sans qu'aucun coupable ne soit désigné.

Le travail de terrain a été mené à Ouahigouya, troisième ville du pays, qui compte 70000 habitants et dans laquelle quatre radios privées opèrent : une associative, une commerciale et deux religieuses. L'accent a été mis sur la radio du fait de son large accès et de sa diffusion en langues nationales. Les autres médias ont été intégrés parallè-

1 Selon les chiffres communiqués par le Conseil Supérieur de l'Information dans son rapport public de 2004. 
lement dans la recherche à titre de référence. La méthodologie a été la suivante :

- Analyser les programmes des radios de la ville et leur mode de production pour identifier l'offre radiophonique de la ville;

- observer au sein de Ouahigouya la manière de décliner les pratiques démocratiques;

- choisir un événement majeur pour chacune de ces pratiques ( vote présidentiel de 2005 pour la pratique de décision, manifestations contre la vie chère en 2005/2006 pour la pratique de protestation etc...);

- identifier et analyser à chaque fois les acteurs de ces pratiques : les électeurs d'un village pour l'élection de 2005, les syndicalistes pour la protestation etc...;

- par des entretiens qualitatifs envisager les modes d'usages des radios par les acteurs et envisager les autres outils mobilisés ou préférés : la place de la radio dans le choix électoral par une enquête par panel auprès des électeurs du village, l'usage fait par les syndicalistes des radios locales pour leur activité de mobilisation et le relais de leur protestation à un niveau local et national etc...

Les résultats de ce travail sont en cours d'élaboration et devraient être achevés au cours de cette année.

\section{Conclusion}

L'approche en termes d'usages explicitée ici s'est construite en s'inspirant des théories développées par la sociologie des médias non culturalisée. Elle a été envisagée empiriquement dans un contexte africain car elle permettait un regard pertinent sur la démocratie en Afrique. Mais elle se veut générale et offre des possibilités d'être transcrite à son tour dans d'autres contextes socio-culturels. À la suite de Quantin qui évoque le politique en Afrique, nous proposons de «banaliser » l'approche des médias en Afrique afin d'ouvrir ce champ de la recherche à la comparaison et surtout de lui permettre de se nourrir théoriquement. Les enjeux et les difficultés d'une telle convergence sont nombreuses. Elle remet aussi en cause une répartition académique par aire culturelle très prégnante. Elle offre cependant la possibilité de proposer des analyses plus systématiques des médias en Afrique à même de rendre compte des véritables enjeux qui se jouent aujourd'hui au sein de la 
sphère publique africaine. Si des études empiriques se développent dans le cadre de cette approche plus généraliste, l'étude des médias en Afrique permettra peut être un jour de dynamiser à son tour la sociologie des médias dans son ensemble.

\section{Bibliographie}

Amérique Latine et Communications. Hermès, 28,(2000) Rubrique Varia, pp. 207270.

Adjovi E. (2003, Déc.). Liberté de Presse et Affairisme Médiatique au Bénin. Politique Africaine, $\mathrm{n}^{\circ}$ 92, pp.157-172.

Althusser Louis (1970). Idéologie et appareils idéologiques d'État. La Pensée, 151, 3-38.

Ang I (1990). The nature of the audience. In Downing J. (Ed.), Questionning the media : a critical introduction. USA.

Balima Serge-Théophile (2000). Les modes et les systèmes d'information publique dans les communes au Burkina Faso. Hermes, n²8, pp.219-231.

Barber Benjamin (1997). La démocratie forte. Paris : Desclée de Brouwer.

Cerfaï Daniel; Pasquier, Dominique. (2003). Les sens du public : Publics politiques, publics médiatiques. Paris : PUF.

Champagne Patrick (1995). La publicisation des opinions. In Pailliart Isabelle (Ed.), L'espace public et l'emprise de la communication. Grenoble : ELLUG.

De la Brosse Renaud (1999). Le Rôle de la Presse écrite dans la transition démocratique en Afrique. PAris : P.U. de Septentrion.

- (2002). Quelques pistes de réflexion sur le rôle des médias dans les transitions démocratiques : analyse comparée des écrits portant sur l'Europe Centrale et Orientale, l'Afrique, l'Amérique Latine et l'Asie. In De la Brosse R.; Horga, I. (Ed.), The Role of the masse media and of the news information and communication technologies in the democratisation process of central and esatern European Societies. Bruxelles : International institute of administrative studies.

Dewey J. (1927). The Public and its Problems. New York : Henry Holt.

Eliasoph N. (2003). Publics fragiles. In Cerfaï D.; Pasquier, D. (Ed.), Les sens $d u$ Public. Paris : Puf.

Frère M-S (2000). Presse et démocratie en Afrique francophone. Les mots et les maux de la transition au Bénin et au Niger. Paris : Karthala.

Hermet Guy (1993). Culture et développement. Paris : Unesco, Albin Michel.

Howard Ross. (2005). Journalistes et Conflits : débats théoriques et actions concrètes. In Frère M.F. (Ed.), Afrique centrale : Médias et Conflits : vecteurs de guerre ou acteurs de paix (pp. 15-48). Bruxelles : GRIP.

Hyden G.; Leslie, M. (2002). Communications and Democratization in Africa, Media and Democracy in Africa (pp. pp.1-27). Uppsala ( Suède) : Nordiska Africainstitutet. 
Hyden Göran; Okigbo, C. (2002). The Media and the two waves of democracy, Media and democracy in Africa (pp. 29-53). Uppsala (Suède) : Nordiska Africainstitutet.

Katz Elihu; Gurevitch, Michael; Haas,Hadassah (1973). On the use of mass media for important things. American Sociological Review, 38, n², 164-181.

Lerner David (1958). The passing of traditional society: Glencoe.

N'Goran Kwame (2001). A bas la démocratie : Klanba Ed.

Quantin Patrick (2005). Voter en Afrique :quels paradigmes pour quelles comparaisons? In Quantin P. (Ed.), Voter en Afrique : comparaisons et différentiations (pp. 11-39). PAris : Harmattan.

Randall Vicky (1993). The Media and democratisation in the Third World. Third Word Quaterly, 14, n³, 625-646.

Schramm William (1964). Mass Media and National Developpement. Standford : Standford University Press.

Tudesq André-Jean (1998). Journaux et radios en Afrique aux XIXe et XXe siècles. Paris : Groupe de recherche et d'échanges technologiques.

- (2000). Les médias en Afrique subsaharienne. L'espoir et l'illusion : GRER, Articles en ligne www.grer.fr.

Unesco (1991). Rapport Final. Séminaire pour le développement d'une presse africaine indépendante et pluraliste. Windhoek, NAmibie : Unesco. 
